\title{
Estudo experimental com o uso de quetamina associada ou não à lidocaína para anestesia epidural caudal em eqüinos
}

\author{
An experimental study of caudal epidural anesthesia in horses using \\ ketamine associated or not to lidocaine
}

\author{
Rafael DeRossi, ${ }^{*}$ Ignácio Alvarez Gómez de Segura**
}

\begin{abstract}
Resumo
Foram aplicadas, experimentalmente, anestesia epidural caudal com apenas quetamina ou associada à lidocaína para avaliar sua eficiência na espécie eqüina. Atualmente, existem evidências clínicas de interações sinérgicas das ações espinhais entre a combinação de uma variada gama de fármacos. Estas podem evitar, ou ao menos reduzir, as ações neurotóxicas que produzem a aplicação isolada de um dos fármacos, que necessitam para isto doses e/ou concentrações superiores para obter a anestesia desejada. Para a realização deste experimento utilizamos seis cavalos de pesos e idades variadas, com os seguintes tratamentos: Grupo I: solução salina; Grupo II: quetamina (1 mg/kg); Grupo III: lidocaína (0,20 mg/kg); e Grupo IV: a associação de quetamina $(1 \mathrm{mg} / \mathrm{kg}) /$ lidocaína $(0,20 \mathrm{mg} / \mathrm{kg})$. Todos os grupos receberam volumes predeterminados e aplicados por via epidural. Para reduzir a variação na resposta, o mesmo grupo de animais foi utilizado no experimento com um intervalo de uma semana entre as diferentes aplicações. A anestesia foi avaliada mediante a estimulação dolorosa, utilizandose uma agulha 23G, e determinando o grau de resposta em uma escala de dor. Os tempos usados foram: basal e 2, 5, 10, 15 minutos e depois a cada 15 minutos, pós-administração de quetamina, lidocaína e a associação de quetamina/lidocaína, ou do grupo controle. Os resultados indicam que o tempo de anestesia e a eficiência da analgesia produzida pelo grupo quetamina/ lidocaína administrada por via epidural foi superior a administração destas drogas individualmente. Os efeitos cardiorrespiratórios foram mínimos em todos os grupos.
\end{abstract}

Palavras-chave: epidural; quetamina; lidocaína; eqüino.

\begin{abstract}
The efficacy of caudal epidural anesthesia in equines using ketamine only or ketamine associated to lidocaine was evaluated experimentally. There are evidences of synergic interactions of the spinal effects by the combination of a broad range of drugs. These combinations may avoid or, at least, reduce the neurotoxic effects produced by the use of one drug only, which application requires higher doses or/and concentrations in order to obtain the desirable anesthesia. In the present study, six horses of various ages and weights were used. They were divided in groups according to the treatment received, as follows: Group I: saline solution; Group II: ketamine (1,0 mg/kg); Group III: lidocaine (0,20 mg/kg); Group IV: association of ketamine (1,0 mg/kg) and lidocaine $(0,20 \mathrm{mg} / \mathrm{kg})$. All groups received pre-determined volumes, given epidurally. In order to reduce variations of the response to the treatment, the same group was used in intervals of one week between the treatments. The anesthesia was evaluated by pain stimulation with a $23 \mathrm{G}$ needle and the response degree was fitted into a pain scale at the times: basal, 2,5 , 10,15 minutes and at each 15 minutes post epidural application of ketamine, lidocaine, ketamine + lidocaine or saline solution.

The results showed that the association of ketamine to lidocaine provides a longer anesthesia and a more effective analgesia when compared to the administration of any of these drugs separately. The cardiopulmonary effects were minimum in all groups.
\end{abstract}

Keywords: epidural; ketamine; lidocaine; horses.

\section{Introdução}

Os anestésicos locais são as drogas de uso mais freqüente para induzir a anestesia epidural em eqüinos para diagnósticos e procedimentos cirúrgicos. Estes deprimem a condução nervosa das fibras simpáticas, sensitivas e motoras. Durante o periodo anestésico o bloqueio das fibras motoras pode acarretar ataxia e decúbito indesejado (Lumb e Jones, 1984), e sua possível toxicidade em doses clínicas pode levar ao aparecimento da síndrome da cauda eqüina e sintomas neurológicos transitórios (Hodgson et al., 1999). Outro efeito destas substâncias é a vasodilatação, por estímulo simpático direto, com conseqüente diminuição no tempo de ação por uma maior absorção (LeBlanc et al., 1990), podendo de-

\footnotetext{
* Professor Adjunto de Técnica Cirúrgica e Anestesiologia, Departamento de Medicina Veterinária, UFMS.

** Pesquisador, Cirurgia experimental - Hospital La Paz - Madrid - Espanha.
} 
terminar hipotensão em animais hipovolêmicos (Morikawa et al., 1974; Nolte et al., 1983).

A administração epidural de quetamina produz anestesia em várias espécies animais (Brock -Utne et al., 1982; Yaksh e Reddy, 1981; Yaksh, 1985; DeRossi, 1996) e no homem (Mankowitz et al., 1982; Bion, 1984). Este efeito parece estar mediado pelos receptores $\mathrm{N}$-metil-D-aspartato (NMDA), que está relacionado com a transmissão e modulação da informação nociceptiva e são bloqueados pelas aricilciclohexaminas como a quetamina (Anis et al., 1983; Thomson et al., 1985). Alguns autores observaram uma possível ação da quetamina pela estimulação de receptores diferentes aos do NMDA. Este efeito a nível local e o mecanismo de ação baseiam-se na hiperpolarização do nervo e depressão do seu potencial de ação, mediados pela estimulação dos receptores opiáceos d (Lee e Frank, 1989), diminuindo a permeabilidade da membrana aos íons sódio e potássio (Baha e Malbert, 1991).

A quetamina, um antagonista dos receptores NMDA é comumente usada por via parenteral para induzir anestesia geral em eqüinos. Produz um estado anestésico dissociativo entre o tálamo-cortical e o sistema límbico. Este estado cataleptóide é representado por nistagmo, manutenção de alguns reflexos, hipertonia caracterizada por movimentos bruscos durante a anestesia e vocalização. A quetamina epidural caudal em eqüinos resulta em anestesia/analgesia, sem produzir efeitos colaterais importantes, como ataxia e depressão cardiorrespiratória (DeRossi, 1996; DeRossi et al., 1998).

O propósito deste estudo é avaliar a anestesia produzida pela associação quetamina/lidocaína por via epidural em eqüinos. A combinação de fármacos com efeito anestésico/analgésico administrados por via epidural parece representar uma vantagem sobre a administração de cada uma delas de forma isolada. O efeito sinérgico produzido pela combinação de fármacos acentua seus efeitos analgésico/anestésico, permitindo a utilização de menores doses e reduzindo seus efeitos secundários (Justin et al., 1982; Niv et al., 1986; Grubb e Riebold, 1992).

\section{Material e métodos}

Para a execução deste experimento, utilizaram-se seis cavalos sem raça definida, sendo três machos e três fêmeas, com peso compreendido entre 310 e 420 quilos, que foram mantidos em baias durante o período do estudo.

Tabela 1 - Animais avaliados

\begin{tabular}{cccccc}
\hline Cavalo & $\begin{array}{c}\text { Idade } \\
(\mathbf{a n o s})\end{array}$ & $\begin{array}{c}\text { Peso } \\
(\mathbf{k g})\end{array}$ & $\begin{array}{c}\text { Sexo } \\
(*)\end{array}$ & $\begin{array}{c}\text { Volume } \\
\text { Administ rado (m) }\end{array}$ & $\begin{array}{c}\text { Comprimento do } \\
\text { cateter (cm) }\end{array}$ \\
\hline 1 & 3 & 310 & M & 7,5 & 14 \\
2 & 3,5 & 350 & M & 8,0 & 15 \\
3 & 16 & 370 & F & 8,3 & 15 \\
4 & 9 & 400 & F & 8,6 & 17 \\
5 & 14 & 420 & F & 9,0 & 18 \\
6 & 6 & 370 & M & 8,5 & 15 \\
\hline
\end{tabular}

(*) $M=$ macho; $F=$ fêmea
Para reduzir a variação na resposta, o mesmo grupo de animais foi utilizado para todos os tratamentos: Grupo I - solução salina; Grupo II - quetamina (1 mg/kg); Grupo III - lidocaína $(0,20 \mathrm{mg} / \mathrm{kg})$, e Grupo IV - quetamina/lidocaína (1 mg/kg + $0,20 \mathrm{mg} / \mathrm{kg}$ ). Cada administração epidural foi realizada com um intervalo mínimo de uma semana para reduzir a influência de um experimento sobre o seguinte.

A quetamina utilizada no experimento foi a forma racêmica, pura e livre de preservativos (Sigmaâ, Aldrich Quimica, S.A. 5 gr, K2753), pesada para cada animal em uma balança de precisão (Precisa 600-Câ) e diluída em solução salina $0,9 \%$.

Para maior comodidade na introdução dos cateteres vasculares a artéria carótida esquerda (Skarda, 1991) foi exteriorizada cinco semanas antes do início do período experimental, segundo técnica modificada de Tavernor (1969).

Esta via foi utilizada durante a fase de experimentação para a medida das pressões arteriais e para a retirada de amostras sangüíneas para gasometria, $\mathrm{pH}$, hematócrito e concentração total de sólidos.

Um cateter epidural (Minibandeja epidural,' com agulha 17G, filtro plano 0,22 micra e cateter epidural com fiador) foi implantado no espaço epidural correspondente à área das vértebras coccígeas Co1-Co2, dois dias anteriores ao início do experimento, permanecendo fixo e reduzindo, desta forma, riscos associados a injeções repetidas. A região foi tricotomizada e preparada cirurgicamente. Os animais foram sedados com xilazina (Rompunâ) ${ }^{2}$ na dose de $1,1 \mathrm{mg} / \mathrm{kg}$, e um botão anestésico foi aplicado na área de implantação do cateter com lidocaína a $2 \% .^{3} \mathrm{O}$ espaço foi identificado através do movimento de cauda (para cima e para baixo), enquanto era localizada através da palpação a depressão entre as duas primeiras vértebras coccígeas. Um trocater epidural de uso contínuo foi introduzido nesta região até sentir o ligamento interarcuato entre as vértebras. Após traspassar este ligamento com o trocater, injetavam-se $4-5 \mathrm{ml}$ de ar e na ausência de resistência, o cateter era inserido no espaço epidural e retirado seu fiador metálico flexível. Sua posição era confirmada com a aplicação de $4-5 \mathrm{ml}$ de lidocaína a $1 \%$. Posteriormente, o cateter foi fixado à pele com cola, ${ }^{4}$ mantendo-se fixo para todas as aplicações do experimento.

Para a administração da anestesia epidural o cateter foi introduzido $12 \mathrm{~cm}$ desde o ponto de inserção na pele, chegando até S3 onde foram depositadas distintas doses de quetamina, lidocaína, quetamina/lidocaína e do grupo controle (solução salina).

Previamente ao início do experimento, foi implantado na artéria carótida esquerda um cateter de 1,2 mm/18G conectado a uma torneira de três vias para tirar amostras sangüíneas arteriais. O cateter também estava conectado a um prolongador de polietileno ligado a um monitor que realizava a mensuração da freqüência cardíaca e das pressões sistólica, diastólica e média.

\footnotetext{
Vygonâ - Valencia, Espanha.

2 Rompun - Bayer do Brasil S.A.

${ }^{3}$ Xilocaína $2 \%$ - Astra-Merrel Lepetit.

${ }^{4}$ Super-Bonder - Loctiteâ.
}

(1)

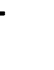


A analgesia/anestesia foi comprovada, por uma mesma pessoa, em todo o estudo, através do estímulo doloroso com uma agulha de $23 \mathrm{G}$ na pele e musculatura profunda do períneo e garupa, seguindo uma escala de três pontos (Gómez de Segura et al., 1998):

$(+++)$ sensação normal, forte resposta ao estímulo doloroso (3);

$(-+)$ ligeira analgesia, resposta deprimida ao estímulo doloroso (2); e

( - ) analgesia profunda, ausência de resposta ao estímulo doloroso (1).

A coleta dos valores após administração dos fármacos ocorreu desde o final da administração epidural (minuto 0) e nos minutos 2, 5, 10, 15, 30, 45, 60, 75, 90, 105 e 120. Obtiveramse os parâmetros anteriormente indicados durante um período mínimo de 90 minutos ou até que o efeito analgésico tivesse desaparecido.

Determinaram-se os valores da média e erro-padrão da média (SEM) para cada um dos grupos estudados. Todos os grupos foram analisados mediante análise de variância (ANOVA), realizando-se, posteriormente, uma comparação entre cada um dos tempos com respeito a seu valor basal, através do teste de Fisher. Considerou-se um valor alfa $=0,05$. O programa utilizado foi o informático Statview (VS 4.0 Abacus Conceptos Inc., Califórnia, USA).

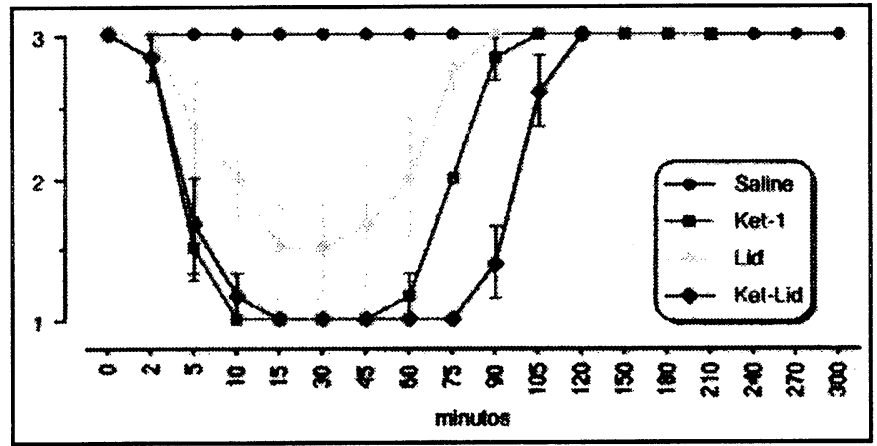

Gráfico 1 - Escala de dor - Períneo (1- Insensibilidade total; 2Analgesia, e 3- sensação normal)

\section{Resultados}

O tempo de analgesia produzido pela combinação quetamina/ lidocaína, administrada pela via epidural, nas três regiões estudadas foi superior a administração destes fármacos individualmente.

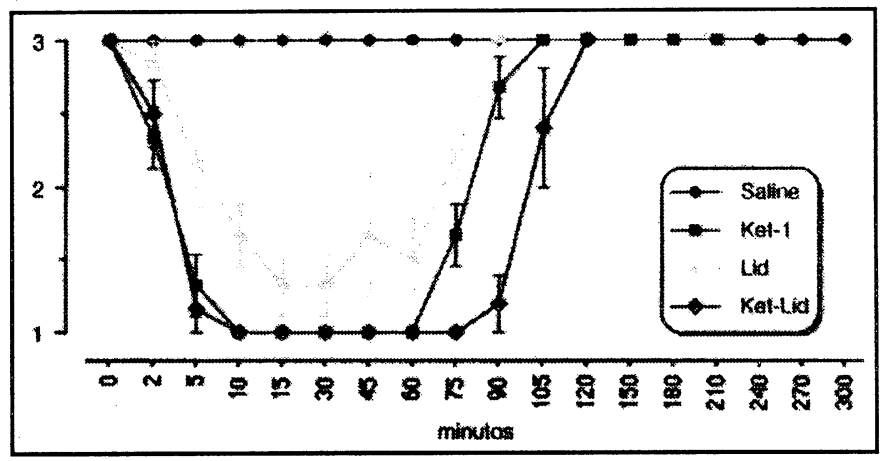

Gráfico 2 - Escala de dor - Cauda (1- Insensibilidade total; 2Analgesia, e 3- sensação normal)

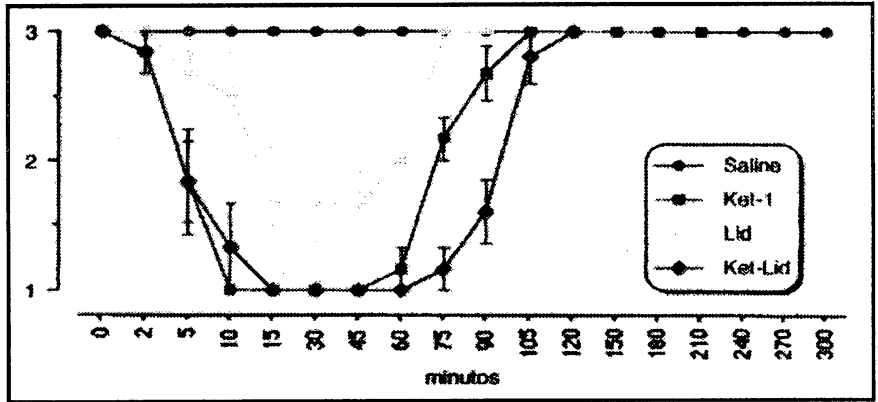

Gráfico 3 - Escala de dor - Garupa (1 - Insensibilidade total; 2 Analgesia, e 3 - sensação normal)

A duração média da analgesia no grupo que recebeu quetamina foi de $60 \pm 15$ minutos; o grupo que recebeu lidocaína não produziu uma analgesia/anestesia efetiva na dose administrada $(0,20 \mathrm{mg} / \mathrm{kg})$ e o grupo que recebeu a combinação quetamina/lidocaína teve em média uma duração de 90 minutos.

Observou-se sonolência, com abaixamento da cabeça em quatro animais (cavalos 1, 2, 4 e 5) após a administração da combinação quetamina/lidocaína do minuto 15 ao minuto 60 .

Quanto aos parâmetros hemodinâmicos, não houve variação significativa na freqüência cardíaca e nas pressões sistólica, diastólica e média (Tabela 2). 
Observou-se sonolência, com abaixamento da cabeça em quatro animais (cavalos 1, 2, 4 e 5) após a administração da combinação quetamina/lidocaína do minuto 15 ao minuto 60 .

Quanto aos parâmetros hemodinâmicos, não houve variação significativa na freqüência cardíaca e nas pressões sistólica, diastólica e média (Tabela 2).

Tabela 2 - Valores cardiovascu-lares e respiratório em seis eqüinos após administração epidural de quetamina (1 mg/kg), lidocaína $(0,20 \mathrm{mg} / \mathrm{kg})$ e quetamina/lidocaína $(1 \mathrm{mg} / \mathrm{kg}+0,20 \mathrm{mg} / \mathrm{kg})$.

\begin{tabular}{|c|c|c|c|c|c|c|c|c|c|c|c|}
\hline & \multicolumn{10}{|c|}{ Tempo (min.) } \\
\hline & & 0 & 2 & 5 & 10 & 15 & 30 & 45 & 60 & 75 & 90 \\
\hline PAS & $\begin{array}{c}\text { Salino } \\
\text { Ket } \\
\text { Lid } \\
\text { Ket/Lid } \\
\end{array}$ & $\begin{array}{l}127 \pm 9 \\
128 \pm 5 \\
123 \pm 3 \\
125 \pm 2 \\
\end{array}$ & $\begin{array}{l}132 \pm 9 \\
131 \pm 5 \\
123 \pm 1 \\
125 \pm 3 \\
\end{array}$ & $\begin{array}{l}134 \pm 9 \\
137 \pm 6 \\
122 \pm 2 \\
124 \pm 2 \\
\end{array}$ & $\begin{array}{l}134 \pm 9 \\
134 \pm 3 \\
122 \pm 4 \\
124 \pm 3 \\
\end{array}$ & $\begin{array}{l}122 \pm 9 \\
145 \pm 7 \\
122 \pm 2 \\
124 \pm 2 \\
\end{array}$ & $\begin{array}{l}116 \pm 8 \\
133 \pm 5 \\
123 \pm 4 \\
125 \pm 3 \\
\end{array}$ & $\begin{array}{l}122 \pm 7 \\
134 \pm 3 \\
125 \pm 4 \\
124 \pm 2 \\
\end{array}$ & $\begin{array}{l}121 \pm 7 \\
128 \pm 6 \\
127 \pm 2 \\
127 \pm 1 \\
\end{array}$ & $\begin{array}{l}121 \pm 8 \\
125 \pm 4 \\
127 \pm 3 \\
125 \pm 3 \\
\end{array}$ & $\begin{array}{l}119 \pm 7 \\
127 \pm 5 \\
123 \pm 4 \\
125 \pm 4\end{array}$ \\
\hline PAM & $\begin{array}{c}\text { Salino } \\
\text { Ket } \\
\text { Lid } \\
\text { Ket/Lid }\end{array}$ & $\begin{array}{c}98 \pm 5 \\
101 \pm 2 \\
93 \pm 4 \\
95 \pm 3 \\
\end{array}$ & $\begin{array}{c}101 \pm 5 \\
99 \pm 2 \\
96 \pm 4 \\
96 \pm 2 \\
\end{array}$ & $\begin{array}{c}103 \pm 5 \\
102 \pm 3 \\
94 \pm 3 \\
95 \pm 2 \\
\end{array}$ & $\begin{array}{c}100 \pm 5 \\
101 \pm 3 \\
95 \pm 2 \\
95 \pm 2 \\
\end{array}$ & $\begin{array}{c}97 \pm 5 \\
103 \pm 3 \\
97 \pm 2 \\
94 \pm 3 \\
\end{array}$ & $\begin{array}{c}93 \pm 4 \\
103 \pm 4 \\
97 \pm 4 \\
96 \pm 4 \\
\end{array}$ & $\begin{array}{c}93 \pm 5 \\
104 \pm 3 \\
95 \pm 2 \\
97 \pm 2 \\
\end{array}$ & $\begin{array}{l}93 \pm 4 \\
97 \pm 3 \\
95 \pm 5 \\
97 \pm 5 \\
\end{array}$ & $\begin{array}{l}92 \pm 5 \\
98 \pm 3 \\
98 \pm 3 \\
95 \pm 3 \\
\end{array}$ & $\begin{array}{r}92 \pm 5 \\
95 \pm 4 \\
98 \pm 2 \\
95 \pm 3 \\
\end{array}$ \\
\hline
\end{tabular}

* grupo quetamina, ${ }^{\star \star}$ grupo lidocaína e ${ }^{\star \star \star}$ grupo quetamina/lidocaína. Abreviações: $(F C)$ - freqüência cardiaca; (FR) - freqüência respiratória; (PAS) - pressão arterial sistólica; (PAD) - pressão arterial diastólica e (PAM) - pressão arterial média.

Não houve diferenças significativas na freqüência respiratória $\left(\mathrm{PaCO}_{2}, \mathrm{PaO}_{2}, \mathrm{HCO}_{3}\right.$ e pH) e de temperatura retal em nenhum dos grupos estudados (Tabela 3 ).

Tabela 3 - pH e parâmetros hemogasimétricos em seis eqüinos após administração epidural de quetamina $(1 \mathrm{mg} / \mathrm{kg})$, lidocaína $(0,20$ $\mathrm{mg} / \mathrm{kg}$ ) e quetamina/lidocaína (1 $\mathrm{mg} / \mathrm{kg}+0,20 \mathrm{mg} / \mathrm{kg})$.

\begin{tabular}{lccccc}
\hline Fármacos & $\begin{array}{c}\text { Tempo } \\
\text { (min.) }\end{array}$ & $\begin{array}{c}\mathbf{P a C O}_{2} \\
(\mathbf{m m H g})\end{array}$ & $\begin{array}{c}\mathbf{P a O}_{2} \\
(\mathbf{m m H g})\end{array}$ & $\begin{array}{c}\mathbf{H C O}_{3} \\
(\mathbf{m m o V})\end{array}$ & $\mathbf{p H}$ \\
\hline Ket.* & Basal & $39,9 \pm 1,5$ & $92,0 \pm 4,1$ & $28,6 \pm 1,7$ & $7,43 \pm 0,01$ \\
& 15 & $38,3 \pm 0,7$ & $94,1 \pm 5,7$ & $26,4 \pm 1,4$ & $7,42 \pm 0,02$ \\
& 30 & $39,6 \pm 1,2$ & $92,4 \pm 4,2$ & $25,1 \pm 1,7$ & $7,42 \pm 0,01$ \\
& 60 & $39,8 \pm 1,5$ & $90,7 \pm 4,6$ & $26,3 \pm 1,8$ & $7,41 \pm 0,07$ \\
& 90 & $41,4 \pm 1,5$ & $90,9 \pm 4,2$ & $26,6 \pm 1,2$ & $7,40 \pm 0,02$ \\
Lid.** & Basal & $38,6 \pm 1,2$ & $94,7 \pm 4,1$ & $25,6 \pm 2,2$ & $7,41 \pm 0,01$ \\
& 15 & $38,8 \pm 1,3$ & $96,3 \pm 4,9$ & $24,3 \pm 1,5$ & $7,40 \pm 0,01$ \\
& 30 & $39,1 \pm 1,1$ & $95,4 \pm 4,1$ & $24,9 \pm 2,0$ & $7,40 \pm 0,02$ \\
& 60 & $39,3 \pm 1,4$ & $96,8 \pm 3,6$ & $24,5 \pm 1,4$ & $7,40 \pm 0,01$ \\
& 90 & $39,8 \pm 1,5$ & $97,1 \pm 5,0$ & $24,1 \pm 1,7$ & $7,39 \pm 0,03$ \\
\hline Ket./Lid.*** & Basal & $40,7 \pm 1,5$ & $95,3 \pm 4,9$ & $28,7 \pm 1,3$ & $7,43 \pm 0,01$ \\
& 15 & $40,1 \pm 1,5$ & $98,2 \pm 4,1$ & $28,8 \pm 1,0$ & $7,43 \pm 0,01$ \\
& 30 & $40,0 \pm 1,0$ & $97,2 \pm 5,1$ & $29,0 \pm 0,9$ & $7,42 \pm 0,01$ \\
& 60 & $39,8 \pm 1,1$ & $98,4 \pm 4,6$ & $29,1 \pm 1,1$ & $7,42 \pm 0,02$ \\
& 90 & $40,2 \pm 1,1$ & $98,2 \pm 4,2$ & $29,4 \pm 0,8$ & $7,41 \pm 0,03$ \\
\hline
\end{tabular}

"grupo quetamina, ${ }^{\star \star}$ grupo lidocaína, ${ }^{\star \star \star}$ grupo quetamina/lidocaína. $(P<0,05)$.
A associação de quetaminallidocaína deste estudo den pociaço de quetamina/lidocaina deste estudo demonstrou possuir um tempo maior de duração da anestesia epidural e qualitativamente melhor do que as duas drogas aplicadas isoladamente nas dosagens usadas. A lidocaína depende da manutenção de uma concentração elevada dentro da fibra nervosa e uma afinidade inerente com o receptor da membrana, para manter um tempo de duração eficiente. Altas dosagens de lidocaína de até $0,45 \mathrm{mg} / \mathrm{kg}$ (Lumb e Jones, 1984; LeBlanc et al., 1988; Grubb et al., 1992) produz um tempo de anestesia mais longo, mas com altas taxas de ataxia e hipotensão. A lidocaína epidural na dosagem de $0,22 \mathrm{mg} / \mathrm{kg}$ produz uma anestesia perineal de $87,2 \pm$ 7,5 minutos de duração (Grubb et al., 1992), sendo que neste experimento foi de $45 \pm 15$ minutos após a administração de $0,20 \mathrm{mg} / \mathrm{kg}$ de lidocaína. A diferença de tempo pode dever-se a diferentes concentrações, ao volume total administrado, ou à utilização do cateter epidural. A aplicação da associação quetamina/lidocaína epidural teve tempo de duração superior de 90 minutos.

É conhecido o bloqueio simpático produzido pelos anestésicos locais epidurais acarretando vasodilatação nas áreas afetadas pelo efeito anestésico, muito embora isto seja clinicamente irrelevante em animais normovolêmicos (Covino e Scott, 1985; 
Perhoniemi e Linko, 1987). Entretanto, em pacientes hipovolêmicos a aplicação destes agentes produzem hipotensão (Morikawa et al., 1974; Nolte et al., 1983). Não há evidências de intervenção das fibras simpáticas no bloqueio epidural com quetamina (Islas et al., 1985), mas alguns autores (Van der Auwera et al., 1987) descrevem casos de hipertensão e taquicardia após cinco minutos da sua aplicação epidural. Nós não observamos nenhuma alteração nas pressões arteriais em nenhum dos grupos estudados (lidocaína, quetamina, quetamina/lidocaína), somente ligeiro aumento não significativo da freqüência cardíaca no grupo quetamina.

Diferentes estudos, incluindo o nosso, não puderam demonstrar alterações da freqüência respiratória, $\mathrm{PaCO}_{2}, \mathrm{PaO}_{2}$ e pH arterial após a administração epidural de anestésicos locais em eqüinos (Skarda e Muir, 1983; Skarda et al., 1984), o mesmo acontecendo com a quetamina (DeRossi, 1996). Outros fármacos aplicados por via epidural, como os agonistas dos receptores a-2 adrenérgicos (LeBlanc e Eberhart, 1990; Skarda e Muir, 1996) e os opiáceos (Zuckerman e Harris, 1994; Brown

\section{Referências}

ANIS, N. A., BERRY, S.C., BURTON, N.R., LODGE, D. The dissociative anaesthetics, ketamine and phencyclidine, selectively reduce excitation of central mammalian neurones by $\mathrm{N}$-methyl-aspartate. $\mathrm{Br}$. J. Pharmacol.v. 79, p. 565-575, 1983.

BAHA, F., MALBERT, C.H. Effect de la kétamine par voie intrathecale chez le chien. Revue Méd. Vét. v. 142, p. 283-285, 1991.

BION, J.F. Intrathecal ketamine for war surgery. A preliminary study under field conditions. Anaesthesiav. 39, p. 1023-1028, 1984.

BROCK-UTNE, J.G., MANKOWITZ, E., KALLICHURUM, S., DOWNING, J.W. Effects of intrathecal saline and ketamine with or without preservative on the spinal nerve roots of monkeys. S. Afr. Med. J.v. 61, p. 360-361, 1982.

BROWN, D.V., McCARTHY, R. J. Epidural and spinal opioids. Anaesthesiology. v. 8, p. 337-341, 1995.

COVINO, B. G., SCOTT, D.B. (Ed.) Handbook of epidural anaesthesia and analgesia. New York: Grune \& Stratton Inc. 1985.

DAVIES, S.N., ALFORD, S.T., COAN, E.J., LESTER, R.A.J., COLLINGRIDGE, G.L. Ketamine blocks na NMDA receptor-mediated component of synaptic transmission in rat hippocampus in a voltagedependent manner. Neurosci. Lett.v. 92, p. 213-217, 1988.

DeROSSI, R. Acción de la ketamina epidural en el caballo (Estudo experimental). 1996. $135 \mathrm{p}$. Tese (Doutorado) - Facultad de Veterinaria - Universidad Complutense de Madrid.

DeROSSI, R., GÓMEZ de SEGURA, I.A., TENDILLO, F., SAN-ROMÁN, J.L. Comparação de quetamina, xilazina e quetamina/xilazina para anestesia epidural em cavalos. In: CONGRESSO BRASILEIRO DE MEDICINA VETERINÁRIA. 25., Gramado, 1997. Anais...p. 66 e 127

GÓMEZ de SEGURA, I.A., DeROSSI, R., SANTOS, M., SAN-ROMÁN, J.L, TENDILLO, F.J. Epidural injection of ketamine for perineal analgesia in the horse. Vet. Surg. v. 27, p. 384-391, 1998.

GRUBB, T.L., RIEBOLD, T.W., HUBER, M.J. Comparison of lidocaine, xylazine, and xylazine/lidocaine for caudal epidural analgesia in horses. J.A.V.M.A.v. 201, p. 1187-1190, 1992.

HODGSON, P.S., NEAL, J.M., POLLOCK, J.E., LIU, S.S. The neurotoxicity of drugs given intrathecally (spinal). Regional Anesthesia and Pain Management, v. 88, p. 787-809, 1999.

ISLAS, J.A., ASTORGA, J., LAREDO, M. Epidural ketamine for control of postoperative pain. Anesth. Analg. v. 64, p. 1161-1162, 1985. e McCarthy, 1995) induzem uma significativa depressão respiratória. A combinação quetamina/lidocaína epidural não apresentou nenhuma alteração respiratória.

A injeção epidural da associação quetamina/lidocaína oferece boa margem de segurança quanto a ataxia e decúbito. A lidocaína epidural na dosagem preconizada de $0,45 \mathrm{mg} / \mathrm{kg}$ (Lumb e Jones, 1984) em eqüinos possui incidência alta de ataxia dos membros posteriores (100\%) (LeBlanc et al., 1988) no entanto estas não são observadas clinicamente, pois grande parte dos animais que recebem este tratamento são mantidos em baias.

Com base em nossos resultados, concluímos que a associação quetamina/lidocaína produz uma efetiva anestesia epidural caudal em eqüinos, com duração mais prolongada, sem nenhuma alteração nos parâmetros cardiovasculares e respiratórios. A ataxia dos membros posteriores acarretada pela lidocaína epidural na dosagem de $0,45 \mathrm{mg} / \mathrm{kg}$, não foi por nós observada nos grupos lidocaína $0,20 \mathrm{mg} / \mathrm{kg}$ e na associação quetamina/lidocaína.

JUSTIN, D.M., FRANCIS, D., HOULTON P.G., REYNOLDS, F. A controlled trial of extradural fentanyl in labour. Br. J. Anaesth.v. 54, p. 409-414, 1982.

LeBLANC, P.H., CARON, J.P. Clinical use of epidural xylazine in the horse. Equine Vet. J.v. 22, n. 3, p. 180-181, 1990.

LeBLANC, P.H., CARON, J.P., PATTERSON, J.S., BROWN, M., MATTA, M.A. Epidural injection of xylazine for perineal analgesia in horses. $J$. Am. Vet. Med. Assoc. v. 193, p. 1405-1408, 1988.

LeBLANC, P.H., EBERHART, S.W. Cardiopulmonary effects of epidurally administered xylazine in the horse. Equine Vet. J. v. 22, n. 6, p. 389391, 1990.

LEE, J.H., FRANK, G.B. Opiod effects of racemic ketamine on the excitability of sciatic nerve and skeletal muscle fibers of the frog. Jpn. J. Pharmacol. v. 51, p. 321-327, 1989.

LUMB, W.V., JONES, E.W. Spinal anesthesia. In: Veterinary Anesthesia. 2. ed., Philadelphia: Lea \& Febiger. 1984.

MANKOWITZ, E., BROCK-UTNE, J.G., COSNETT, J.E., GREENTHOMPSON, R. Epidural ketamine. A preliminary report. S. Afr. Med. J. v. 61, p. 441-442, 1982.

MORIKAWA, K., BONICA, J.J., TUCKER, G.T. Effects of acute hypovolaemia on lignocaine absorption and cardiovascular response following epidural block in dogs. Br. J. Anaesth.v. 46, p. 631-635, 1974. NIV, D., RUDICK, V., GOLAN. A., CHAYEN, M.S. Augmentation of bupivacaine analgesia in labour by epidural morphine. Obstet. Gynecol. v. 67, p. 206-209, 1986.

NOLTE, I., WATNEY, G.C.G., HALL, L.W. Cardiovascular effects of epidural blocks in dogs. J. Small Anim. Pract.v. 24, p. 17-21, 1983.

PERHONIEMI, V., LINKO, K. Effect of spinal versus epidural anaesthesia with $0,5 \%$ bupivacaine on lower limb blood flow. Acta Anaesthesiol. Scand. v. 31, p. 117-121, 1987.

SCLELLING, C.G., KLEIN, L.V. Comparison of carbonated lidocaine and lidocaine hydrochloride for caudal epidural anesthesia in horses. $A m$. J. Vet. Res.v. 46, p. 1375-1377, 1985.

SKARDA, R.T. Local anesthetics and local anesthetic techniques in horses. In: MUIR, W.W., HUBBEL, J.A.E. (ed.) Equine anesthesia. Monitoring and emergency treatment. St. Louis: Mosby Year book, 1991. p. 199-246.

SKARDA, R.T., MUIR, W.W. Analgesic, hemodynamic, and respiratory effects of caudal epidurally administered xylazine hydrochloride solution in mares. Am. J. Vet. Res. v. 57, p. 193-200, 1996. 
SKARDA, R.T., MUIR, W.W. Continous caudal epidural and subarachnoid anesthesia in mares: a comparative study. Am. J. Vet. Res. v. 44, p. 2290-2298, 1983.

SKARDA, R.T., MUIR, W.W., IBRAHIM, A.L. Plasma mepivacaine concentrations after caudal epidural and subarachnoid injection in the horse: Comparative study. Am. J. Vet. Res.v. 45, n.10, p. 1967-1971, 1984.

STODDART, A.P., NICHOLSON, K.E., POPHAM, P.A. Low dose bupivacaine/fentanyl epidural infusions in labour and mode of delivery. Anaesthesiav.49, p. 1087-1090, 1994.

TAVERNOR, W.D. Technique for the subcutaneous relocation of the common carotid artery in the horse. Am. J. Vet. Res. v. 30, p. 18811883, 1969.

THOMSON, A.M., WEST, D.C., LODGE, D. An N-methylaspartate receptor-mediated synapse in rat cerebral cortex: a site of action of ketamine? Nature. v. 313, p. 479-481, 1985.
VALVERDE, A., LITLE, C.B., DYSON, D.H., MOTTER, C.H. Use of epidural morphine to relieve pain in a horse. Can. Vet. J.v. 31, p. 211-212, 1990. VANDER AUWERA, D., VERBORGH, C., CAMU, F. Epidural ketamine for postoperative analgesia. Anesth. Analg. v. 66, p. 1340, 1987.

YAKSH, T.L. Pharmacology of spinal adrenergic systems which modulate spinal nociceptive processing. Pharmacol. Biochem Behav. v. 22, p. 845-858, 1985.

YAKSH, T.L., COLLINS, J.G. Studies in animals should precede human use of spinally administered drugs. Anesthesiologyv. 70, p. 4-6, 1989.

YAKSH, T.L., REDDY, S.V.R. Studies in the primate on the analgesic effects associated with intrathecal actions of opiates, alpha-adrenergic agonist and baclofen. Anesthesiologyv. 54, p. 451-467, 1981.

ZAUGG, J.L., NUSSBAUM, M. Epidural injection of xylazine: a new option for surgical analgesia of the bovine abdomen and udder. Vet. Med.p. 1043-1046, 1990.

ZUCKERMAN, R.L., HARRIS, A.P. Anesthesia in obstetrics. Curr. Opin. in Obstet. and Ginecol. v. 6, p. 408-413, 1994. 